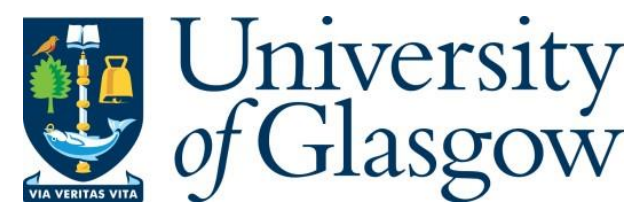

Bird, H. J.A., Ramesh, K., Ōtomo, S. and Maria Viola, I. (2021) Usefulness of inviscid linear unsteady lifting-line theory for viscous large-amplitude problems. AIAA Journal, (doi: 10.2514/1.J060808).

There may be differences between this version and the published version. You are advised to consult the publisher's version if you wish to cite from it.

https://eprints.gla.ac.uk/263058/

Deposited on: 12 January 2022

Enlighten - Research publications by members of the University of Glasgow https://eprints.gla.ac.uk 


\title{
The usefulness of inviscid linear unsteady lifting-line theory for viscous large-amplitude problems
}

\author{
Hugh J. A. Bird, Kiran Ramesh ${ }^{\dagger}$ \\ Aerospace Sciences Division, School of Engineering, University of Glasgow, Glasgow, United Kingdom, G12 8QQ \\ Shūji Ōtomo * and Ignazio Maria Viola ${ }^{\S}$ \\ School of Engineering, Institute for Energy Systems, University of Edinburgh, Edinburgh, United Kingdom, EH9 3BF
}

Unsteady Lifting-Line Theory (ULLT) is a low order method capable of modeling interacting unsteady and finite wing effects at low computational cost. Most formulations of the method assume inviscid flow and small amplitudes. Whilst these assumptions might be suitable for small-amplitude aeroelastic problems at high Reynolds numbers, modern engineering applications increasingly involve lower Reynolds numbers, large amplitude kinematics and vortex structures that lead to aerodynamic non-linearities.

This paper establishes that ULLT still provides a useful solution for low Reynolds number, large-amplitude kinematics problems, by comparing ULLT results against those of experimentally validated computational fluid dynamics simulations at $\mathbf{R e}=10000$. Three-dimensional (3D) effects stabilize Leading Edge Vortex (LEV) structures, resulting in a good prediction of whole wing force coefficients by ULLT. Whilst the inviscid spanwise force distributions are accurate for small-amplitude kinematics, the ULLT cannot model 3D vortical structures, and thus it cannot correctly predict the force distribution due the LEV. It can however predict the shedding of LEVs to a limited extent via the leading edge suction parameter criterion. This can then be used as an indicator of the usefulness of the force distribution results.

\section{Nomenclature}

$$
\begin{array}{ll}
\mathbb{R} & =\text { aspect ratio } \\
c & =\text { chord } \\
C_{l} & =\text { two-dimensional lift coefficient } \\
C_{L} & =\text { three-dimensional lift coefficient } \\
C_{m} & =\text { two-dimensional moment coefficient }
\end{array}
$$

\footnotetext{
*Graduate Researcher, h.bird.1@research.gla.ac.uk, Student Member AIAA

†Senior Lecturer, Aerospace Sciences Division, School of Engineering, Kiran.Ramesh@glasgow.ac.uk, Senior Member AIAA

${ }^{\ddagger}$ Ph.D Student, School of Engineering, Institute for Energy Systems, s.otomo@ed.ac.uk

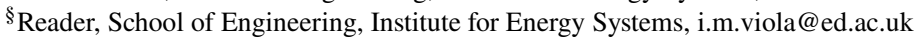




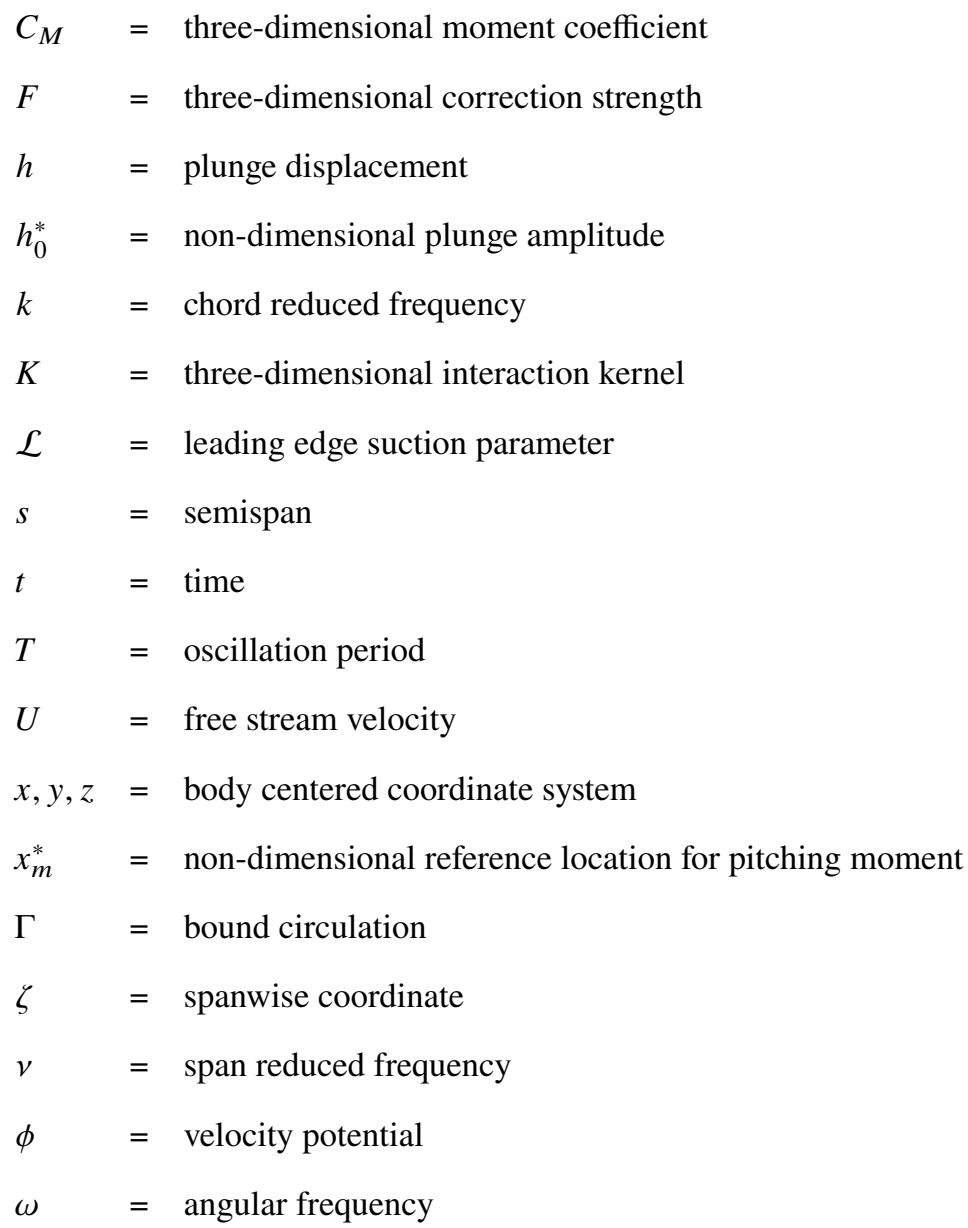

\section{Introduction}

RADITIONALLY, unsteady aerodynamics focused on inviscid, small amplitude problems. This was to reduce the complexity of the problem sufficiently for analytical solutions to be obtained using low-order models. These low-order models discard non-essential elements of the flow physics to make the problem easier to solve. Common models are based on potential flow and assume that the fluid is inviscid and incompressible. Geometric assumptions are often made in order to linearize the problem. To formulate a low order method, the problem's physics must first be understood.

Research on the unsteady aerodynamics of finite wings was initially driven by the problem of dynamic stall in helicopters [1]. Computational Fluid Dynamics (CFD) and experimental results are reviewed by Carr [2], Carr et al. [3] and Ekaterinaris and Platzer [4]. More recent investigations include those of Angulo et al. [5], Visbal and Garmann [6, 7] and Costes et al. [8]. These studies involved oscillating wings in the high Reynolds number, low reduced frequency regime. Modern applications such as micro air vehicles, high altitude long endurance drones and unmanned aerial vehicles have inspired research at lower Reynolds numbers, with greater amplitudes and higher reduced frequencies, 
often involving Leading Edge Vortices (LEVs), trailing edge vortices and wing tip vortices. Such flows are the subject of this article. Studies in this regime have been carried out on translating wings [9-11], pitching wings [12-17], plunging wings [18-23], rotating wings [24-29], and wings subject to gusts [30-32], and have shed light on contributions from circulatory, apparent mass and vortical effects.

In two dimensions (2D), discrete vortex particle methods have proven valuable in low-order modeling. Linear potential flow theories such as Theodorsen [33] assume small-amplitude oscillations and a planar wake. By using discrete vortex particles to model the non-planar wake, Ramesh et al. [34] and Yan et al. [35] considered arbitrarily large kinematics. Criteria for the shedding of LEVs have allowed these models to be extended to include intermittent LEV shedding. The Leading Edge Suction Parameter (LESP) hypothesis [36] states that for a given flow regime, an airfoil can only provide so much suction at the leading edge. If this suction is exceeded, vorticity is shed from the leading edge. This suction was initially linked to the vorticity distribution of a thin airfoil model by Katz [37] and Ramesh [36].

Less progress in low-order modeling has been made in three dimensions (3D). Boundary element methods [38], unsteady vortex lattice methods [39-42] or vortex particle methods [43] can be used. However, these methods lack robust and simple LEV shedding criteria, and have numerical difficulties in modeling unsteady wakes. This shortcoming of 3D methods can be overcome with strip theory [44]. Multiple 2D solutions can be applied to the varying chord distribution of a 3D wing. But this neglects important 3D effects, failing to account for a loss of lift due to induced downwash in low aspect ratio wings, for example. By correcting these multiple unsteady 2D solutions to account for 3D effects, Unsteady Lifting-Line Theory (ULLT) is obtained.

ULLT uses the same ideas as steady lifting-line theory, originally credited to Prandtl [45]. Prandtl combined 2D models with a simplified 3D correction model to correct for finite wing effects. This idea of separating a difficult problem into two simple problems with different length scales was formalized by Van Dyke [46].

ULLT includes additional corrections for variations in vorticity downstream of the wing due to the unsteady nature of the problem. Early work [47, 48] was asymptotically limited to low-frequency oscillation of the wing. Further work [49, 50] extended the frequency range before Guermond and Sellier [51] produced a method suitable for swept wings at any oscillation frequency. Research in the time domain has been less extensive, and asymptotically limited by the assumptions made in modeling the wake [52-57]. A more detailed review can be found in Bird and Ramesh [58].

These ULLT are all based upon potential-flow theory. They assume that the flow is inviscid and incompressible. They also all assume that vorticity is only shed from the trailing edge of the wing, and cannot model LEVs. However, in practice, the theoretical limitations of a model can belie its applicability.

For LLT, this has been demonstrated repeatedly: the asymptotic limitations of the method mean that it is not strictly applicable to elliptic or rectangular wings, as explained by Van Dyke [46]. In practice, it provides good predictions. However, doing so may require discretization to avoid problems with wingtip downwash singularities. In ULLT, Bird and Ramesh [58] found that simplified wake models were often sufficient to obtain a good solution. And in 2D, McGowan 
et al. [59] found that Theodorsen's method could provide good force-coefficient predictions even in the low Reynolds number, chord reduced frequency of $O(1)$ regime, and in the presence of LEV shedding when examining the problem of pitch-heave cancellation. In this regime, there is negligible separation of the boundary layer near the trailing edge, and the flow detaches directly at the leading edge, forming a leading edge vortex. Similar results for more complex kinematics can be found in Elfering and Granlund [60], and for finite wings in Bird and Ramesh [61].

This paper aims to explore the limits of applicability of unsteady lifting-line theory when applied to low Reynolds number flows and large-amplitude kinematics. Two dimensional low order models that assume inviscid flow have frequently been successful for finding lift and moment coefficients in the low Reynolds number regime, and ULLT is the natural 3D extension of these. Verifying that ULLT formulated for inviscid problems can be applied to such cases is a stepping stone to the problem of accounting for LEV shedding.

An unsteady lifting-line theory, based on Sclavounos' method [50, 58], will be applied to oscillating heave problems in the low Reynolds number regime $(R e=10000)$ for wings of aspect ratios 6, 3 and 1. Low amplitude, LEV-free cases will be examined along with larger amplitude cases. Through comparison with experimentally validated, Unsteady Reynolds Averaged Navier-Stokes (URANS) CFD results, the extent to which ULLT is usable to obtain forces and force distributions on a wing will be established, and a new concept of applying the LESP criterion to predict LEV shedding using ULLT on finite wings will be explored.

A short introduction to Sclavounos' ULLT is given in Sec. II] along with the means to obtain both force and leading-edge suction distributions in Sec. [I.A and Sec. II.B respectively. This is followed by a discussion of the method's soon to be violated theoretical limitations in Sec. II.C. Next, the applicability of the ULLT is explored through comparison to CFD in Sec. III] The test cases, the CFD and the validation of the CFD are detailed in Sec. III.A Sec. III.B and Sec. III.C. This then allows the whole wing forces and LESP distributions to be examined in Sec. III.D. along with the form of the LEVs at the wing center in Sec. III.E. Finally, the force distributions will be compared in Sec. III.F. Conclusions are made in Sec.IV

\section{Theoretical approach}

In this section we present an unsteady lifting-line theory, based on the work of Sclavounos [50]. Here, we consider pure plunge oscillations. For a more detailed derivation including pitching kinematics, see Bird and Ramesh [58].

This ULLT considers a wing in inviscid, incompressible flow undergoing small amplitude oscillation, as shown in Fig. 1. The freestream velocity of the flow is $U$ in the positive $x$ direction, and the plunge displacement of the wing is

$$
h(y ; t)=h_{0} e^{i \omega t}=h_{0}^{*}(y) c(y) e^{i \omega t},
$$

where $h$ is the plunge displacement, $h_{0}$ the plunge amplitude, and $h_{0}^{*}$ is the plunge amplitude non-dimensionalized by 


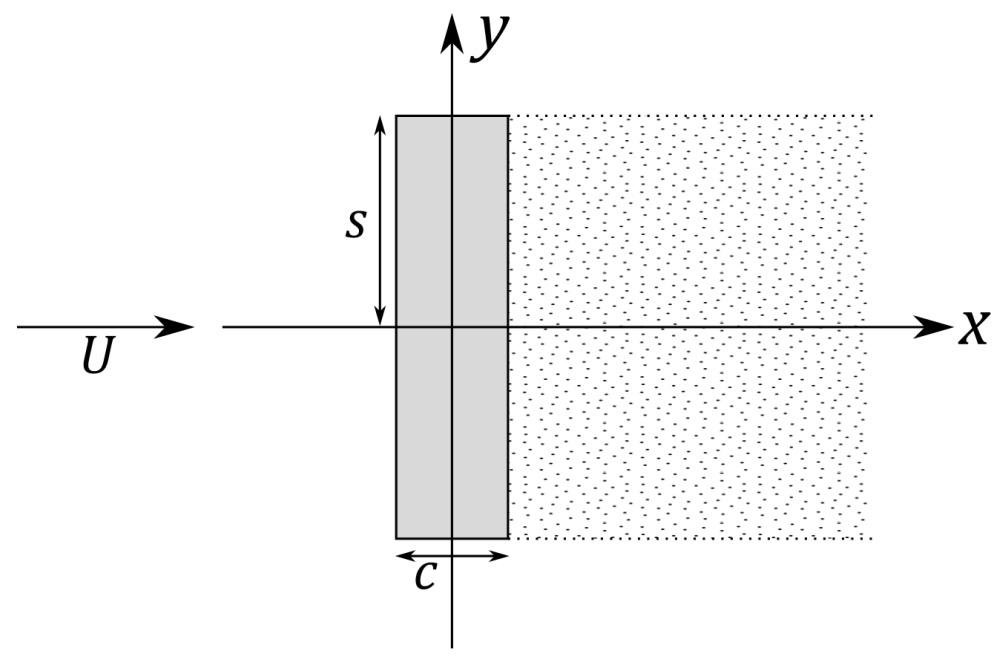

Fig. 1 The rectangular wing and its wake.

the chord $c$. The spanwise coordinate is $y, t$ is time, and $\omega$ is the angular frequency of oscillation. The frequency of oscillation can be non-dimensionalized either with respect to chord or span as

$$
k(y)=\frac{\omega c(y)}{2 U}, \quad v=\frac{\omega s}{U},
$$

where $k$ is referred as chord reduced frequency and $v$ is the span reduced frequency based on the semispan $s$.

Lifting-line theory is based upon the separation of length scales. For a wing of high aspect ratio, the chord scale is much smaller than the span scale. This means that the detail of the chord scale problem can be neglected in the span scale problem. And if the chord scale problem changes slowly with respect to span, it can be modeled as a 2D problem with a 3D correction.

Here, the velocity potential $\phi(x, y, z ; t)$ derived as a $2 \mathrm{D}$ solution with a $3 \mathrm{D}$ correction is given as

$$
\phi(x, y, z ; t) \approx \phi^{2 D}(x, z ; t)+F(y)\left(i \omega z e^{i \omega t}-\phi_{n}^{2 D}(x, z ; t)\right)
$$

where the first term $\phi^{2 D}(x, z ; t)$ represents the velocity potential solution to the $2 \mathrm{D}$ problem (which is identical to Theodorsen's problem). The second term represents the 3D 'correction' to the $2 \mathrm{D}$ problem. Here, this is modeled by an oscillating uniform downwash $F(y) i \omega z e^{i \omega t}$, and the reaction of the $2 \mathrm{D}$ section $F(y) \phi_{n}^{2 D}(x, z ; t)$ to this, with the subscript $n$ in $\phi_{n}^{2 D}$ signifying a unit heave amplitude. The $F(y)$ term represents the complex amplitude of this correction with respect to span, and $\phi_{n}^{2 D}(x, z ; t)$ the $2 \mathrm{D}$ velocity potential solution of a section oscillating in heave with unit amplitude.

Since $\phi^{2 D}$ is equivalent to Theodorsen's problem it allows the $2 \mathrm{D}$ bound circulation $\Gamma^{2 D}$, to be obtained: 


$$
\Gamma^{2 D}(y ; t)=\frac{4 U h_{0}^{*}(y) c(y) e^{-i k}}{i H_{0}^{(2)}(k)+H_{1}^{(2)}(k)} e^{i \omega t},
$$

where $H_{0}^{(2)}(k)$ and $H_{1}^{(2)}(k)$ are Hankel functions of the second kind.

Since the system is linear with respect to amplitude, this allows corrected distributions to be found as

$$
\Gamma(y ; t)=\Gamma^{2 D}(y ; t)-F(y) \Gamma_{n}^{2 D}(t) .
$$

The 3D correction strength $F(y)$ is given by Sclavounos as

$$
F(y)=-\frac{1}{2 \pi i \omega e^{i \omega t}} \int_{-s}^{s} \Gamma^{\prime}(\eta) K(y-\eta) \mathrm{d} \eta,
$$

where

$$
K(y)=\frac{1}{2 s} \operatorname{sgn}\left(y^{*}\right)\left[\frac{e^{-v\left|y^{*}\right|}}{\left|y^{*}\right|}-i v E_{1}\left(v\left|y^{*}\right|\right)+v P\left(v\left|y^{*}\right|\right)\right],
$$

where $E_{1}(x)$ is the exponential integral [62], $y^{*}=y / s$ is the normalized span coordinate and

$$
P(y)=\int_{1}^{\infty} e^{-y t}\left[\frac{\sqrt{t^{2}-1}-t}{t}\right] \mathrm{d} t+i \int_{0}^{1} e^{-y t}\left[\frac{\sqrt{1-t^{2}}-1}{t}\right] \mathrm{d} t .
$$

Substituting this into Eq. 5 gives a differential equation

$$
\Gamma(y ; t)-\frac{\Gamma_{n}^{2 D}(t)}{2 \pi i \omega e^{i \omega t}} \int_{-s}^{s} \Gamma^{\prime}(\eta) K(y-\eta) \mathrm{d} \eta=\Gamma^{2 D}(y, t),
$$

where an approximate solution can be obtained by taking the bound vorticity distribution as a Fourier series

$$
\Gamma(y ; t)=4 U s \sum_{m=1}^{M} \Gamma_{m} \sin (m \zeta) e^{i \omega t},
$$

where $y=-s \cos \zeta$. This is solved at collocation points distributed over the span. 


\section{A. Obtaining lift and moment coefficients}

As with the bound vorticity, Theodorsen's theory [33] allows the lift and moment coefficients associated with $\phi^{2 D}$ to be found as

$$
\begin{aligned}
& C_{l}^{2 D}(y ; t)=2 \pi h_{0}^{*}\left(-2 i k C(k)+k^{2}\right) e^{i \omega t}, \\
& C_{m}^{2 D}(y ; t)=2 \pi h_{0}^{*}\left[-2 i k C(k)\left(x_{m}^{*}-\frac{1}{4}\right)+k^{2}\left(x_{m}^{*}-\frac{1}{2}\right)\right] e^{i \omega t},
\end{aligned}
$$

where $C(k)=\frac{K_{1}(i k)}{K_{1}(i k)+K_{0}(i k)}$ is Theodorsen's function in terms of modified Bessel functions of the second kind [62] and $x_{m}^{*}$ is the moment reference location in $[0,1]$, with 0 being the leading edge and 1 the trailing edge.

These can be corrected for 3D effects as

$$
\begin{aligned}
C_{l}(y ; t) & =C_{l}^{2 D}(y, t)-F(y) C_{l n}^{2 D}(y), \\
C_{m}(y ; t) & =C_{m}^{2 D}(y, t)-F(y) C_{m n}^{2 D}(y) .
\end{aligned}
$$

For the whole wing, this allow lift and moment coefficients to be found as

$$
C_{L}=\frac{L}{\rho U^{2} s \bar{c}}=\frac{1}{2 s \bar{c}} \int_{-s}^{s} C_{l}(y) c(y) \mathrm{d} y, \quad C_{M}=\frac{M}{\rho U^{2} s \bar{c}^{2}}=\frac{1}{2 s \bar{c}^{2}} \int_{-s}^{s} C_{m}(y) c^{2}(y) \mathrm{d} y,
$$

where $\bar{c}$ is the mean chord of the wing.

\section{B. Leading edge suction and criticality}

Recently, the idea of the leading edge suction parameter (LESP) [36] has become popular as a means by which to include the shedding of a leading edge vortex in discrete-vortex enhanced thin-airfoil theories.

At an airfoil's rounded leading edge, suction is required for the flow to remain attached when going around it. The LESP is a measure of this suction, and is calculated from the singular leading-edge term $A_{0}$ in thin-airfoil theory. For a given aerodynamic regime, it has been found that there is a maximum LESP that can be supported by the rounded leading-edge shape. When this critical LESP is exceeded, flow separation occurs and a shear layer is shed from the leading edge, forming a leading edge vortex [36, 63].

For finite wings, Hirato [64] found that the LESP criterion still applied, with LEV formation on any 2D section occurring as predicted by the section's leading edge suction. In their work, the LESP at wing section was numerically calculated using the unsteady vortex lattice method. In this research, we obtain an analytical expression for the 3D LESP from the ULLT.

The inner solution of the current method is based upon Theodorsen's method. Following Ramesh [65], the leading 
edge suction parameter $\mathcal{L}(y)$ can be extracted from Theodorsen's problem:

$$
\mathcal{L}^{2 D}(y ; t)=-2 i k h_{0}^{*} C(k) e^{i \omega t} .
$$

This can then be corrected for 3D effects in the same manner as $\Gamma, C_{l}$ and $C_{m}$.

$$
\mathcal{L}(y ; t)=\mathcal{L}^{2 D}(y, t)-F(y) \mathcal{L}_{n}^{2 D}(y, t) .
$$

When $\mathcal{L}$ exceeds a certain critical value $\mathcal{L}_{\text {crit }}$ at any spanwise location, it is expected that vorticity will be shed from the leading edge at that section. This value $\mathcal{L}_{\text {crit }}$ must be obtained empirically. When the critical LESP value is exceeded, we expect that the attached flow assumption made in the derivation of the ULLT will have been violated.

\section{Theoretical limitations}

Sclavounos' unsteady lifting-line theory is subject to theoretical limitations both as a result of the potential flow model used, and the asymptotic assumptions of lifting-line theory. The derivation of both Theodorsen and Sclavounos' theories assume inviscid, incompressible flow, a planar wake and small amplitude oscillations. It is assumed that vorticity is shed from only the trailing edge of the wing.

Both lifting-line theory and the thin-airfoil model upon which Theodorsen's theory is based, are asymptotic methods. The airfoil section is assumed to be thin, and the wing is assumed to be of high aspect ratio, with the inner (2D) solutions required to change slowly with respect to span, in order to preserve the two-dimensionality of the inner solution problems. Sclavounos also assumes that the downwash induced by the outer domain on the inner domain is uniform. This limits the asymptotic validity of the method to where $k$ is much less than one.

In practice, users of lifting-line theory have routinely ignored some of these theoretical limitations. Square and elliptical tipped wings have an inner solution that changes too rapidly with respect to span near the tip to be valid. Whilst some error is introduced near the tips, an integro-differential approach to solving the lifting-line problem gives a usable result in practice.

\section{Results}

In this section, unsteady lifting-line theory is compared to results obtained from Reynolds-Averaged Navier-Stokes (RANS) CFD for the case of a rectangular heaving wing. Details of the cases studied are given in Sec. III.A. Then, the CFD method is described in Sec. III.B. The CFD is validated against experiment in Sec. III.C Results for whole wing forces, wing center vorticity slices and lift distributions from both CFD and ULLT are compared in Sec. III.D, Sec. III.E and Sec. III.F respectively. 


\section{Table 1 Case parameters}

\begin{tabular}{ll}
\hline Parameter & Values \\
\hline$k$ & 0.4 \\
$R$ & $1,3,6, \infty$ \\
$h_{0}^{*}$ & $0.05,0.5,1$ \\
\hline
\end{tabular}

\section{A. Comparison cases}

For all cases, a rectangular wing with a NACA0008 airfoil section and squared off wing tips is studied. The wing is oscillating in heave, according to the kinematics given by Eq.11 The Reynolds number is 10000 and the chord reduced frequency is $k=0.4$. The chord reduced frequency is chosen in the intermediate range since it is expected to produce the most challenging LEV-dominated flows for the ULLT to predict. At this frequency, 3D effects remain strong and the forces are not dominated by added mass effects [58]. At high frequencies, added mass effects dominate the forces reducing the $3 \mathrm{D}$ effects [58], and at low frequencies strong and coherent LEVs are not expected to form.

The wing is oscillating in plunge with one of three amplitudes: $h_{0}^{*}=0.05$, a small amplitude where leading edge vortex shedding is not expected; $h_{0}^{*}=0.5$, an amplitude where LEV shedding is expected, and $h_{0}^{*}=1$, a larger amplitude still. Wings of aspect ratio 6, 3 and 1 were examined along with the 2D case $(\boldsymbol{R}=\infty)$ for comparison. These parameters are summarized in Table 1

\section{B. Numerical methods}

URANS 3D computations of unsteady fluid dynamics are performed at Reynolds number of 10000 using the open-source CFD toolbox OpenFOAM [66]. The choice of URANS for this study allows the exploration of a larger parameter space than would be possible with LES. In all flows investigated in this article, separation occurs directly at the leading edge, rather than progressing from trailing edge to leading edge (as in classical dynamic stall). In these scenarios, URANS has previously been shown to be capable of accurately predicting the formation, convection and detachment of LEVs, and the forces and moment on the wing [36, 63, 64].

A body-fitted computational mesh is moved in accordance with prescribed rate laws, and the time-dependent incompressible Navier-Stokes are solved using a finite-volume method. A second-order backward implicit scheme is adopted to discretize the transient terms, while second-order, limited Gaussian integration schemes are used for the gradient, divergence and Laplacian terms. The pressure implicit with splitting of operators (PISO) algorithm is employed to achieve pressure-velocity coupling. The Spalart-Allmaras (SA) turbulence model [67] is used for turbulence closure. The SA model is chosen for this problem because of extensive previous experience in applying it successfully for unsteady, separated and vortex-dominated flows at $R e=10000$ such as those considered in this research [36, 59]. The trip terms in the original SA model are turned off, and for the low Reynolds number cases considered in this 


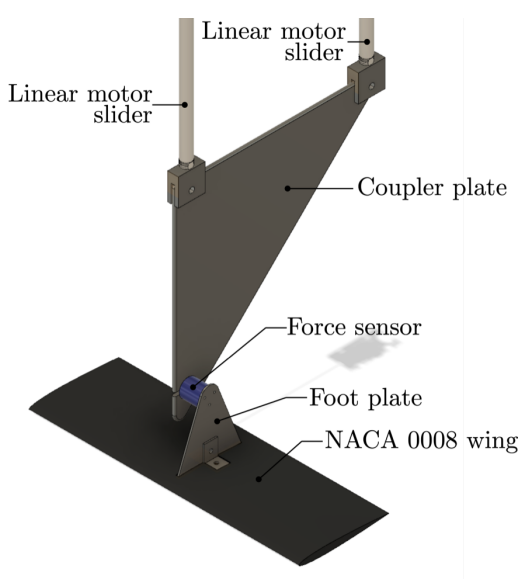

(a) Detailed view

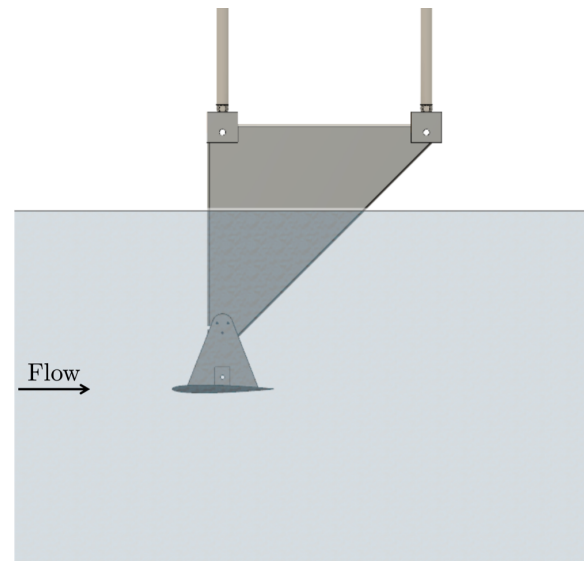

(b) Side view

Fig. 2 Test section and experimental setup

research, the effects of the turbulence model are confined to the shed vortical structures and wake.

The chord length $c=0.1 \mathrm{~m}$. The O-mesh has 116 cells chordwise, with increased resolution near the leading and trailing edges. There are 211,105 and 35 cells in the spanwise direction for aspect ratios 6, 3 and 1 respectively. The spanwise domain extends 4 chord lengths beyond the wingtip with an average spacing of 21 cells per chord length in this region. In the wall-normal direction, cell spacing begins at $1.5 \times 10^{-5} \mathrm{~m}$ next to the wall $\left(y^{+}<1\right)$ and extends a distance of 11.5 chord lengths away from the wing with an average density of 16.3 cells per chord length. The simulations were carried out at a free stream velocity $U=0.1 \mathrm{~m} / \mathrm{s}$ and kinematic viscosity $10^{-6} \mathrm{~m}^{2} / \mathrm{s}$.

\section{Validation of CFD against experiment}

The CFD simulations were validated against an experiment performed in the water flume at the University of Edinburgh. A wing with a NACA0008 airfoil section, a chord length $c=0.1 \mathrm{~m}$ and an aspect ratio of $\boldsymbol{R}=3$ was 3D printed. The freestream velocity was fixed at $0.1 \mathrm{~m} / \mathrm{s}$, resulting in the chord based Reynolds number of 10000 . The plunging foil rig consists of two linear motors (LinMot, PS01-23x80F-HP-R20) connected with each other via a linkage system and a coupler plate. This is shown in Fig. 2 .

Direct force measurements are conducted. A six-axis force/torque sensor (ATI Inc., Nano-17 IP68) is mounted between the coupler plate and the foot plate. The sensor is capable of measuring forces in the plane of the wing cross section up to $\pm 25 \mathrm{~N}$, and $\pm 35 \mathrm{~N}$ in the orthogonal direction, and moments up to $\pm 250 \mathrm{~N} \mathrm{~m}$ around the three axes with a resolution of $1 / 160 \mathrm{~N}$ for the forces and $1 / 32 \mathrm{Nm}$ for the moments. LabVIEW is used to trigger the prescribed motor kinematics through a digital output device and also to start recording forces for a synchronized measurement through a DAQ board. A sampling frequency of $10 \mathrm{kHz}$ is used to record the forces, which are then filtered in three steps. Firstly, a fourth-order Butterworth low-pass filter with a cutting frequency of $75 \mathrm{~Hz}$ was applied. Then the data was smoothed with a 200 points moving average. The last step is a sixth-order Chebyshev II low-pass filter with $-20 \mathrm{~dB}$ attenuation in 


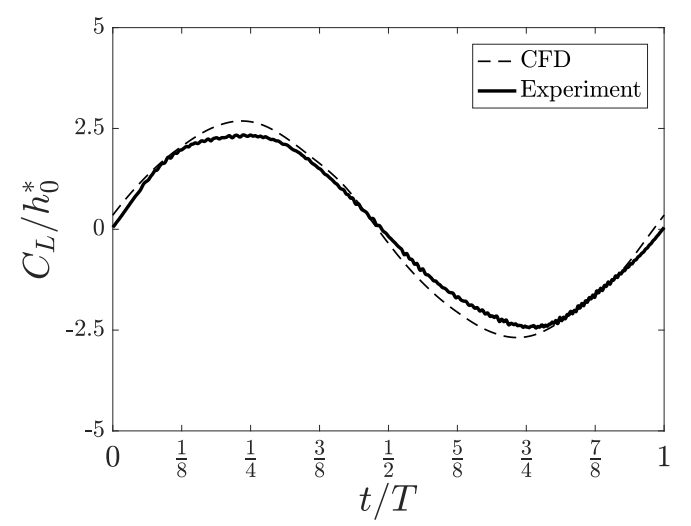

Fig. 3 Comparison of the lift coefficients obtained from CFD and experiment for a plunging rectangular aspect-ratio-three wing for $h_{0}^{*}=0.5$ and $k=0.4$.

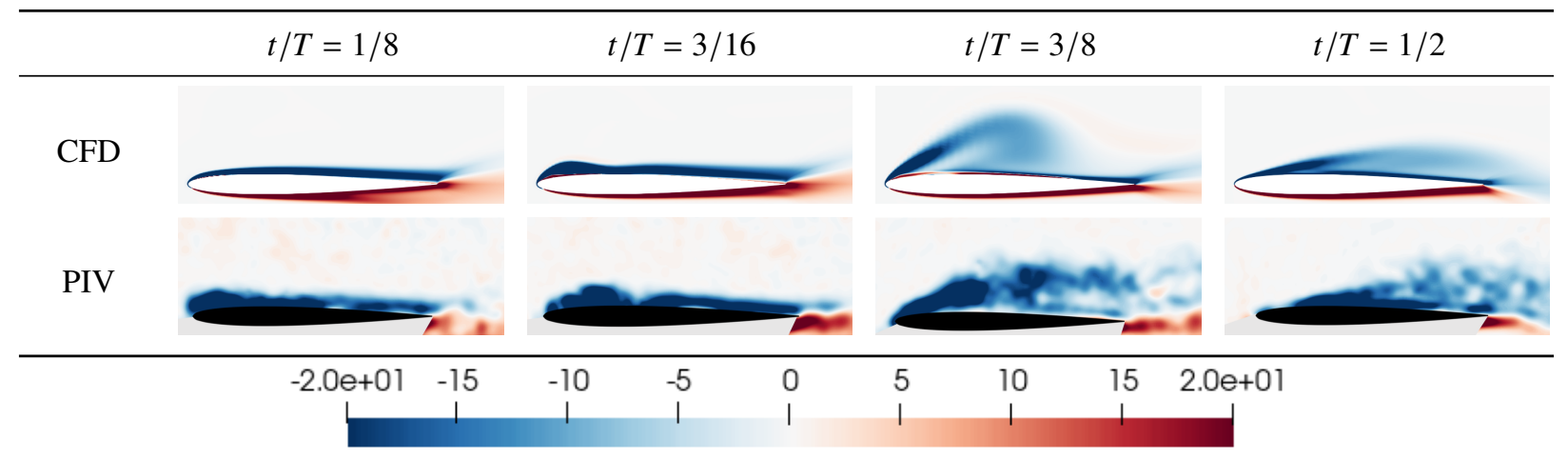

Fig. 4 Comparison of experimental and CFD quarter span vorticity distributions for rectangular aspect-ratiothree wings oscillating in heave at $k=0.4$ and $h_{0}^{*}=0.5$.

the stopband. This three-step filtering method can preserve load spikes. Phase-averaging is applied for 20 periods.

Particle image velocimetry (PIV) is used to perform flowfield analysis . A double pulsed Nd:YAG laser (New Wave Research, Solo PIV, $532 \mathrm{~nm}, 200 \mathrm{~mJ}$ ) is used to illuminate the plane at 1/4 of the span of the wing, with silver coated hollow glass spheres (Potters Industries, $10 \mu \mathrm{m}$ ) used as seeding. Images are then obtained by a CCD camera (IMPERX, B2020 equipped with Nikon $50 \mathrm{~mm}$ lens) with a resolution of 2056 pix $\times 2060$ pix. Velocity vectors are computed using adaptive multi-pass cross-correlation, with a first interrogation window of 64 pix $\times 64$ pix, and a final interrogation window of 32 pix $\times 32$ pix, and an overlap of 50\% (DaVis, LaVision Inc.). Phase averaging is undertaken over 30 periods of Gaussian filtered PIV data.

The validation case was that of the aspect-ratio-three wing oscillating at amplitude $h_{0}^{*}=0.5$ and $k=0.4$. This amplitude is sufficient to cause the formation of leading edge vortices. A comparison of the lift coefficients obtained from the CFD and the experiment are shown in Fig. 3. PIV data is compared at $t / T=[1 / 8,3 / 16,3 / 8,1 / 2]$ - critical points that demonstrate the development of LEV at the quarter span on the wing. This is shown in Fig. 4.

The CFD and experiment are in good agreement for the lift coefficients. The flow visualization from PIV data 


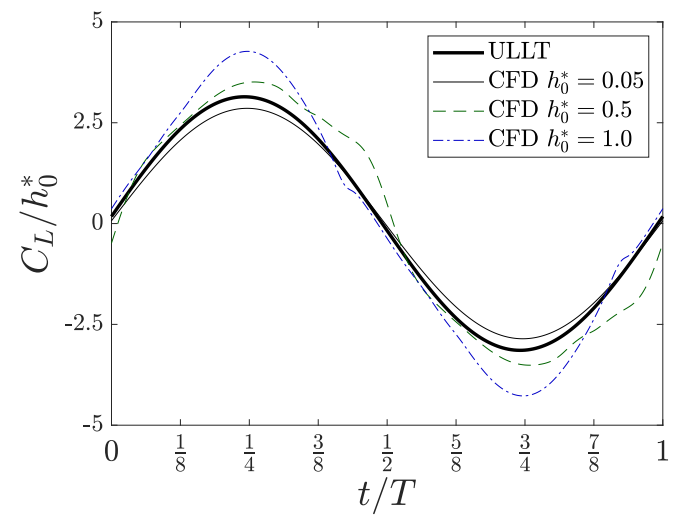

(a) $2 \mathrm{D}$

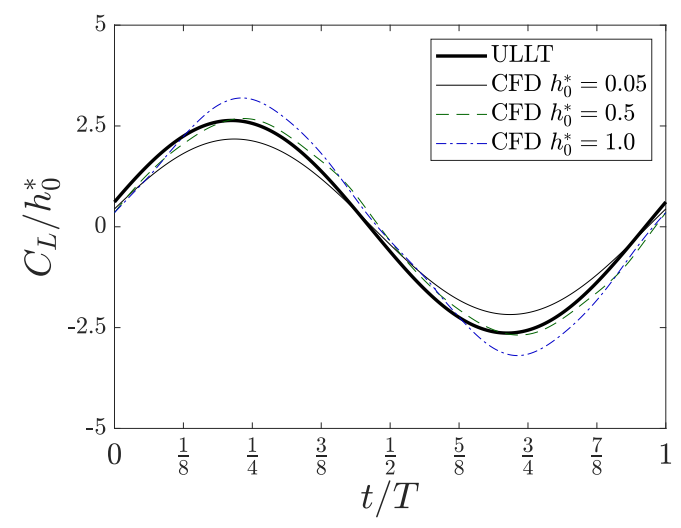

(c) Aspect ratio 3

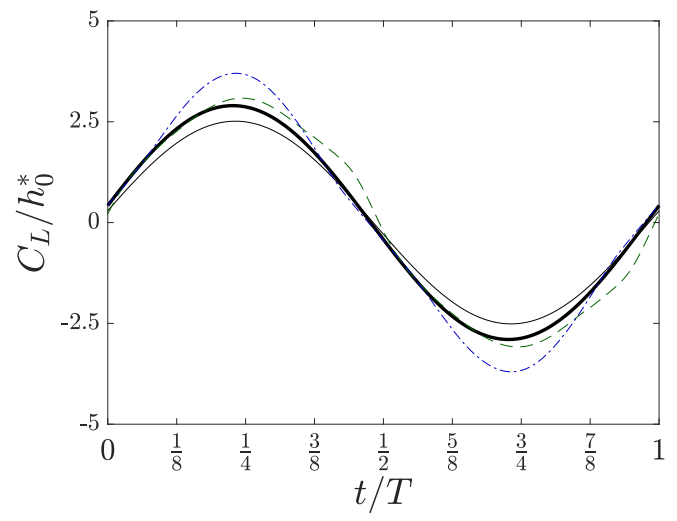

(b) Aspect ratio 6

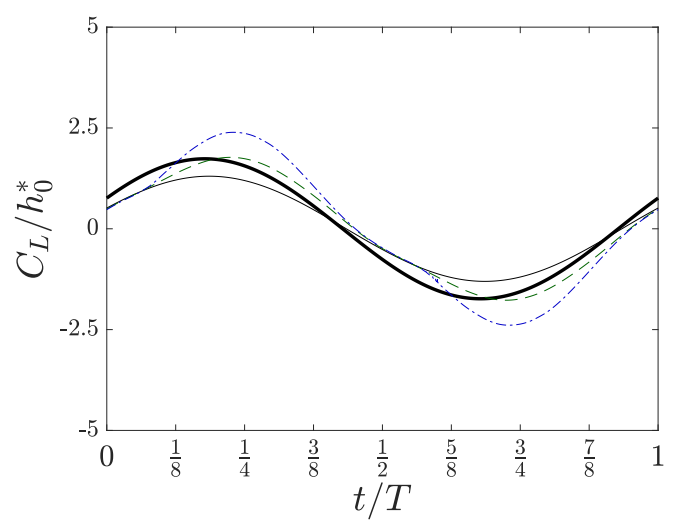

(d) Aspect ratio 1

Fig. 5 Comparison of the predicted lift coefficients of ULLT and CFD for rectangular wings oscillating in heave at various amplitudes and aspect ratios at $k=0.4$.

also agrees well with that from the CFD. Both methods show the formation of the initialization of LEV formation between $t / T=1 / 8$ and $t / T=3 / 16$. At $t / T=3 / 8$, the angle of the leading edge shear layer matches, along with the approximate shape of the LEV region. By $t / T=1 / 2$, the methods both show that the vortex structure has been convected downstream, and remains attached to the surface of the airfoil.

\section{Lift and moment coefficient comparison}

Figure 5 shows a comparison of whole wing lift coefficients for the cases listed in Table 1 . The lift coefficient predicted by Sclavounos' ULLT varies linearly with oscillation amplitude, so lift coefficients have been normalized by oscillation amplitude to allow for better comparison.

The 2D problem, equivalent to an infinite aspect ratio wing, is shown in Fig. 5(a) At low amplitude $h_{0}^{*}=0.05$, the CFD result is sinusoidal in form. As oscillation amplitude increases to $h_{0}^{*}=0.5$ and then $h_{0}^{*}=1$, the peak lift increases super-linearly with respect to oscillation amplitude. This is a result of the leading-edge vortex being formed at these amplitudes. At the intermediate amplitude $h_{0}^{*}=0.5$, the LEV is both shed later than in the $h_{0}^{*}=1$ case, and is slower to 


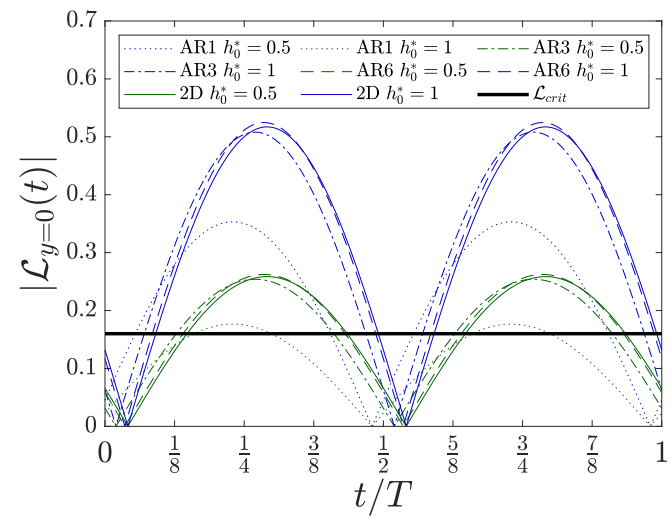

(a) LESP against time at wing center

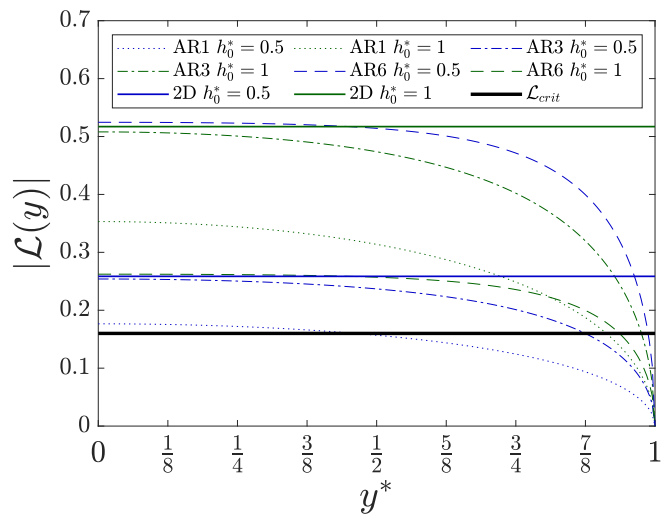

(b) LESP amplitude spanwise distribution

Fig. 6 Comparison of the predicted leading edge suction parameter of ULLT for rectangular wings oscillating in heave at various amplitudes and aspect ratios at $k=0.4$.

pinch off from the airfoil. As a consequence, the lift enhancement comes later (visible in the humped shape of the $C_{L}$ curve). This LEV shedding can be linked to the LESP criterion. The leading-edge suction of the 2D cases will be examined before the results of the finite aspect ratio cases are analyzed.

The ULLT / Theodorsen predicted value of LESP from Eq. 17] is shown in Fig. 6] and can be used to predict this LEV shedding. The time-varying value of the leading edge suction parameter at the wing center $\mathcal{L}_{y=0}$ is shown in Fig. 6(a) Leading-edge vortex formation occurs when the critical value of LESP $\mathcal{L}_{\text {crit }}$ is exceeded. The critical value $\mathcal{L}_{\text {crit }}$ was found by calibration to be 0.16 using the method described in Ramesh et al. [36]. This model predicts that LEV shedding will occur at all aspect ratios, for both $h_{0}^{*}=0.5$ and $h_{0}^{*}=1.0$. The LESP values for the $h_{0}^{*}=0.05$ cases are not plotted since they are small and do not approach the critical value of LESP. Comparison between Fig. 6(a) and Fig. 51does yield a relationship. However, the flow around an airfoil for oscillating kinematics depends on historical LEV shedding. Since this ULLT does not model LEVs, this means that it is unable to account for this. Consequently, it is more useful to focus on the amplitude of the oscillating LESP value, as presented in Fig. 6(b) In 2D, the values of $\mathcal{L}$ obtained using Theodorsen's theory predict the LEV formation that occurs in the CFD results. Additionally, the larger value of predicted LESP amplitude that occurs as the amplitude of the kinematics increases suggests the formation of a stronger LEV. Consequently, the LEV has a greater impact on the force coefficients.

Unsteady lifting-line theory, equivalent to Theodorsen's theory at infinitely high aspect ratio, predicts both the phase and amplitude of the smallest amplitude case well. The higher amplitude cases, $h_{0}^{*}=0.5$ and $h_{0}^{*}=1$, have a non-sinusoidal $C_{L}$ waveform, unlike that predicted by the theory. Nevertheless, given the difference in flow field, explored in Sec. III.E and Sec. III.F the accuracy with which the CFD result can be predicted is reasonably good.

Figures 5(b) 5(c) and 5(d) show the results of ULLT compared to CFD for decreasing aspect ratio.

As would be expected, the CFD shows that the lower aspect ratio wings produce less lift. This is due to the downwash 


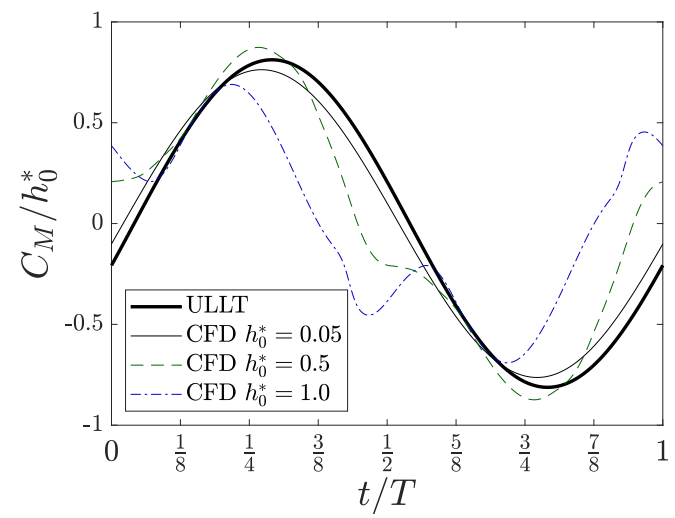

(a) $2 \mathrm{D}$

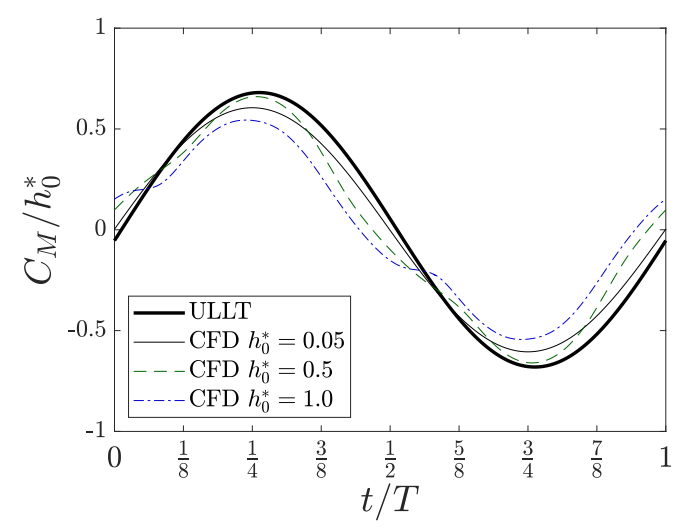

(c) Aspect ratio 3

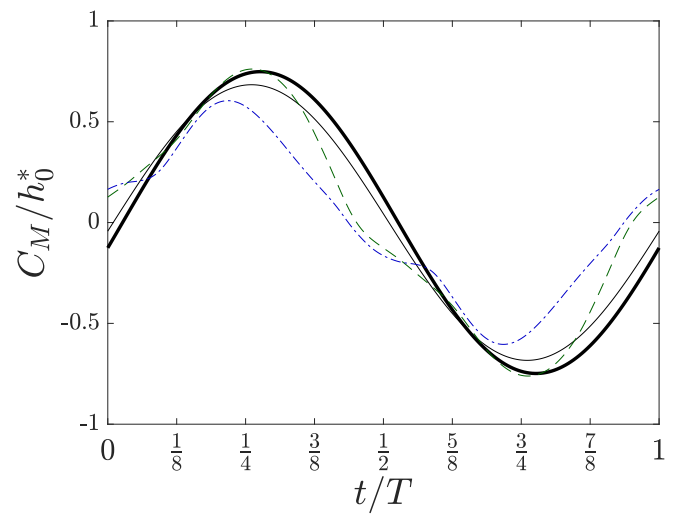

(b) Aspect ratio 6

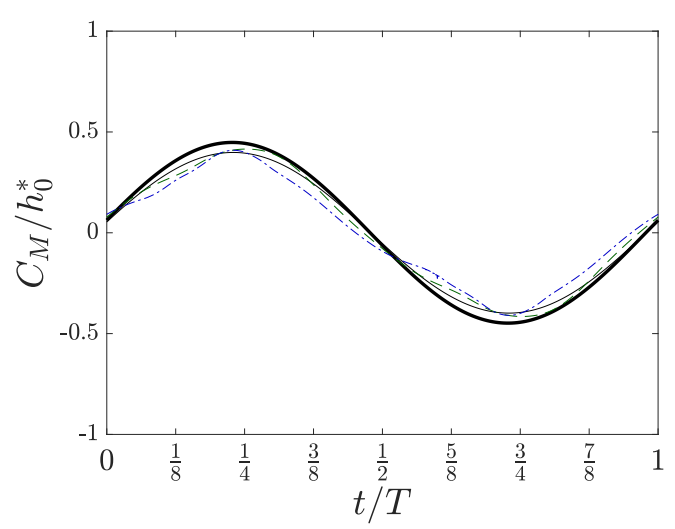

(d) Aspect ratio 1

Fig. 7 Comparison of the predicted mid-chord moment coefficients of ULLT and CFD for rectangular wings oscillating in heave at various amplitudes and aspect ratios at $k=0.4$.

induced by the trailing wing tip vortices resulting in a lower effective plunge amplitude $\left(h_{0}(y)-F(y)\right)$ than found in the 2D case. This effect cannot be predicted by strip theory. And at all aspect ratios the lift continues to vary super-linearly with amplitude. Increasing the amplitude disproportionately increases lift due to the effects of the leading edge vortex. This cannot be predicted by the linearized ULLT used here.

Focusing on the result obtained by unsteady lifting-line theory for amplitude $h_{0}^{*}=0.05$, it predicts both phase and amplitude well at aspect ratio 6, and the error isn't significantly larger than the difference between the Theodorsen prediction and the 2D CFD result. As aspect ratio decreases from $A 6$ to $R 3$ to $R 1$, the ULLT slightly over-predicts the amplitude of $C_{L}$, although the sinusoidal waveform assumption and phase prediction remained good. For the higher oscillation amplitudes, correctly predicted LEV formation occurs, and consequently the $C_{L}$ waveform from the CFD becomes non-sinusoidal. However, the normalized amplitude is still predicted reasonably well by the inviscid ULLT. For the cases with LEV shedding, the difference between CFD and ULLT becomes smaller as $\mathbb{R}$ decreases.

The whole wing moment coefficients are shown in Fig. 7 The 2D case $C_{M}$ curve is shown in Fig. 7(a), The low-amplitude heave kinematics results in a sinusoidal $C_{M}$ curve. For the $h_{0}^{*}=0.5$ case the normalized $C_{M}$ is initially 
similar to that of the low-amplitude case. As the LEV develops and sheds, the $C_{M}$ initially increases and then drops markedly, resulting in a non-sinusoidal wave. The $C_{M}$ briefly returns to the same normalized amplitude as the low amplitude case before another LEV forms during the second half of the cycle. The $h_{0}^{*}=1$ case shows the same trends but with LEV initiation happening earlier and the subsequent loss of $C_{M}$ being greater. As the aspect ratio decreases, the high amplitude results obtained from the CFD tend to the low amplitude $h_{0}^{*}=0.05$ case. Both the initial LEV-produced increase and the subsequent reduction in $C_{M}$ during LEV shedding are diminished by finite wing effects.

At all aspect ratios the ULLT predicts the $C_{M}$ results of the low amplitude $h_{0}^{*}=0.05$ cases with good accuracy in term of both amplitude and phase. The ULLT cannot predict the non-sinusoidal $C_{M}$ curves resulting from the large amplitude cases at high aspect ratio, although the amplitude and phase are approximately correct. The dwindling effects of the LEV on $C_{M}$ as aspect ratio decreases results in the ULLT providing a better prediction at lower aspect ratio.

For both the lift and moment results, as aspect ratio decreases the impact of the LEV decreases and the waveform becomes more sinusoidal. Whilst ULLT cannot model LEVs, the LESP obtained from ULLT can be used to predict LEV formation. It predicts that larger amplitudes will result in a larger value of LESP. This is reflected in the super-linear relationship between $C_{L}$ and amplitude at all aspect ratios. For $C_{M}$, increased values of LESP correspond to a larger deviation from the sinusoidal waveform produced by low amplitude results. As aspect ratio decreases this deviation from sinusoidal reduces, despite the fact that LEV formation is still predicted by ULLT. In the next section (Sec. III.E), the form of the LEV obtained from CFD is examined more closely.

\section{E. Wing center vorticity distribution}

The spanwise component of vorticity at the center of the wings has been plotted in Fig. 8 for amplitudes $h_{0}^{*}=0.5$ and $h_{0}^{*}=1$. The low amplitude cases with $h_{0}^{*}=0.05$ are not shown since they did not produces leading edge vortices, as predicted by ULLT.

For both amplitudes, the aspect ratio 6 case provides results similar to $2 \mathrm{D}$. For the $h_{0}^{*}=0.5$ case, at $t / T=1 / 16$ and $t / T=1 / 8$ the boundary layer on the wing's upper surface is attached. As the plunge stroke continues, the plunge velocity increases and, consequently, a greater leading edge suction is required in order for the flow to remain attached around the leading edge. At $t / T=3 / 16$, an attached leading-edge vortex has formed. As the downstroke continues, the leading edge vortex becomes larger, and is convected downstream. At $t / T=3 / 8$ the downstroke velocity is reducing, but the main vortex structure remains in close proximity to the airfoil surface. The shear layer feeding the LEV becomes a prominent feature. At $t / T=1 / 2$, the downstroke has finished. The LEV has now been convected to the trailing edge and vorticity has spread out due to viscous effects. The shear layer from the leading edge remains attached, but has weakened.

At the higher amplitude $h_{0}^{*}=1$ the process is initially similar, but starts earlier due to the increased velocity of the downstroke. An attached LEV has formed by $t / T=1 / 8$. By $t / T=3 / 16$, the LEV has grown. A large counter rotating 


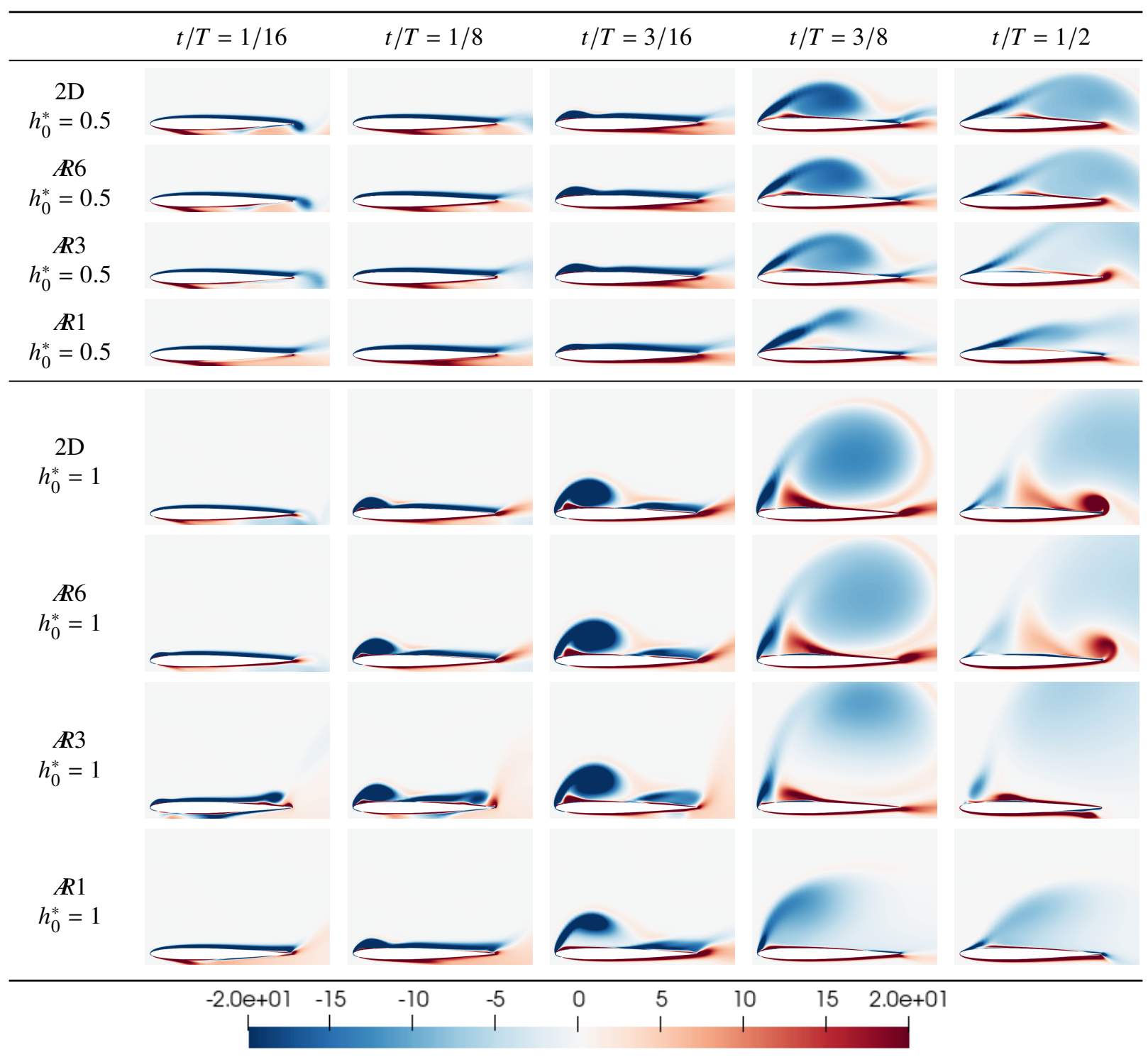

Fig. 8 Wing center spanwise vorticity distributions for rectangular wings oscillating in heave at $k=0.4$. The in-plane vorticity is normalized as $\omega^{*}=\omega_{y} \bar{c} / U$ 
region of vorticity has been drawn under the LEV. This region has grown significantly by $t / T=3 / 8$ and has separated the LEV from the wing surface. The LEV is now larger and more diffused, but also at some distance from the wing surface. By $t / T=1 / 2$, the LEV has completely detached from the shear layer that initially fed it. It induces a counter vortex at the trailing edge.

3D effects allow the LEV to remain attached for a longer period of time, and suppress the formation of the trailing-edge vortex (see Visbal [16]). This effect is most visible for the $h_{0}^{*}=1, \mathbb{R} 1$ case and results in simpler $C_{L}$ and $C_{M}$ curves. For higher aspect ratio wings the LEV at the wing center is similar to that of the $2 \mathrm{D}$ problem. However, near to the wing tip the induced downwash decreases the effective angle of attack leading to a smaller vortex (see Fig. 6(b)].

In the next section (Sec. [II.F), the spanwise 3D vortex structures and force distributions are examined, and the claim that the impact of the LEV is reduced near the wing-tips substantiated.

\section{F. Spanwise force distributions}

When the assumptions of its derivation are satisfied, ULLT can predict force distributions along the span of wing. Here, we compare the data obtained from ULLT to the data obtained from CFD. The lift and moment distributions for the $h_{0}^{*}=0.05$ amplitude at aspect ratios 1 and 6 are shown in Fig. 9 9 The range of ULLT results over the whole cycle is marked by the shaded region.

Both distributions at both aspect ratios have broadly smooth curves. However, the lift distribution at the very tip of the wing is non-smooth due to separation at the sharp edges of the squared off tips. The overestimation of the force amplitude follows that obtained for whole wing forces found earlier. The phase error remains small. At aspect ratio 6, shown in Fig. 9(a), ULLT predicts the shape of the curves very well. The lift coefficient varies significantly with respect to span only near the wing tip, suggesting that the flow is $2 \mathrm{D}$ over the majority of the wing. For the aspect ratio 1 case (Fig. 9(b)], the prediction is worse. For low Reynolds number, low amplitude cases, ULLT provides a good prediction of force distribution with respect to span.

Figure 10 shows the force distributions for the large amplitude $h_{0}^{*}=1$ cases, along with a visualization of the LEV obtained by taking a $\mathrm{Q}$ criterion [68] iso-surface for $Q=1$. Both the force and vorticity distributions are shown at times $t / T=\{1 / 16,1 / 8,3 / 8,1 / 2\}$. These times correspond to the growth of the $h_{0}^{*}=1$ LEV shown in Fig. 8

At aspect ratio 1, the vortex structures that forms through the sinusoidal stroke results in an increase in forces near the wing tips. At the center of the wing, the $C_{l}$ and $C_{m}$ are similar to that predicted by unsteady lifting-line theory. ULLT combined with the LESP criterion predicted that LEV shedding would occur in these high amplitude cases, as shown in Figure 6 The LESP criterion does correctly predict LEV shedding in the broadest sense at low aspect ratio. Notably, whilst Fig. 6(b) suggests that LEV shedding does not occur near the wing tip, significant vortical structures still exist here. The 2D flow that ULLT assumes at each point on the wing span is evidently incorrect around the prominent vortices at the wing tips. Consequently, the lift distribution obtained from ULLT does not match that from CFD. 


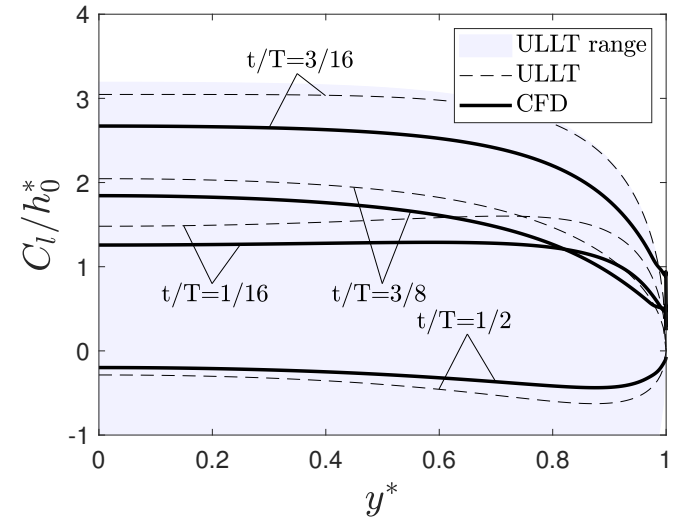

(a) Aspect ratio $6 C_{l}$

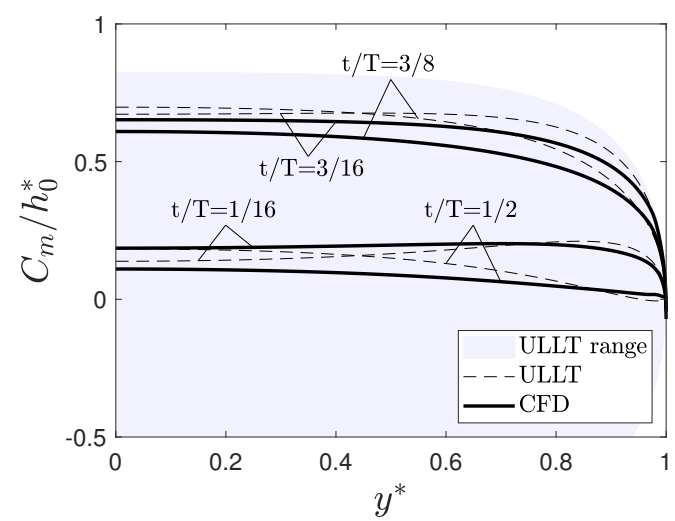

(c) Aspect ratio $6 C_{m}$

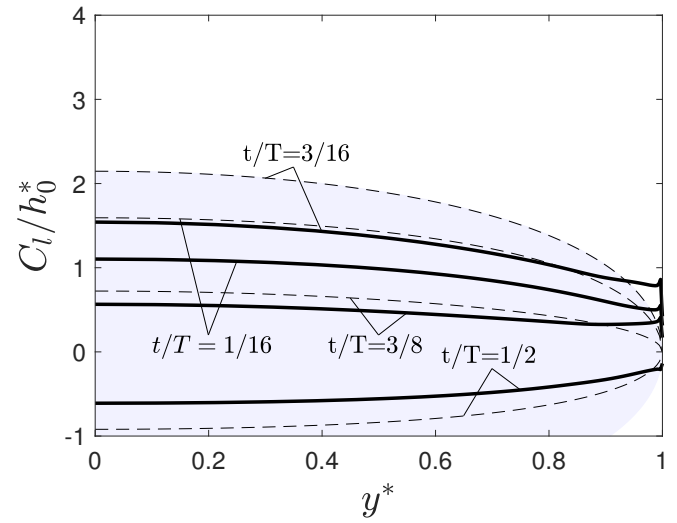

(b) Aspect ratio $1 C_{l}$

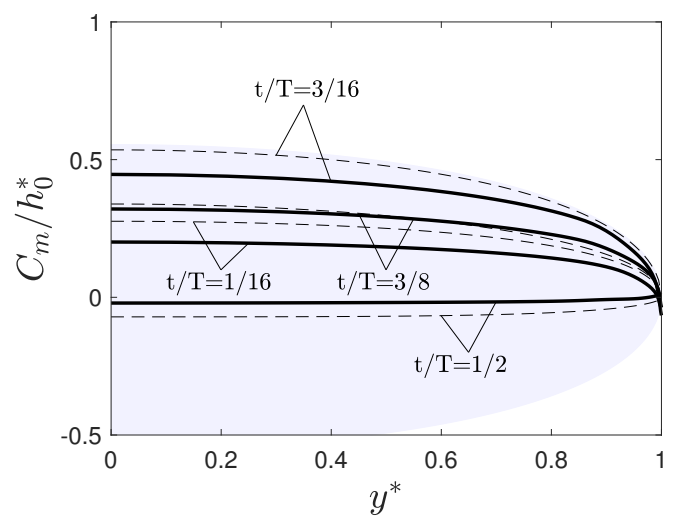

(d) Aspect ratio $1 C_{m}$

Fig. 9 Lift and moment distributions for rectangular wings oscillating in heave with amplitude $h_{0}^{*}=0.05$ at $k=0.4$. Moments are taken about the mid-chord. 


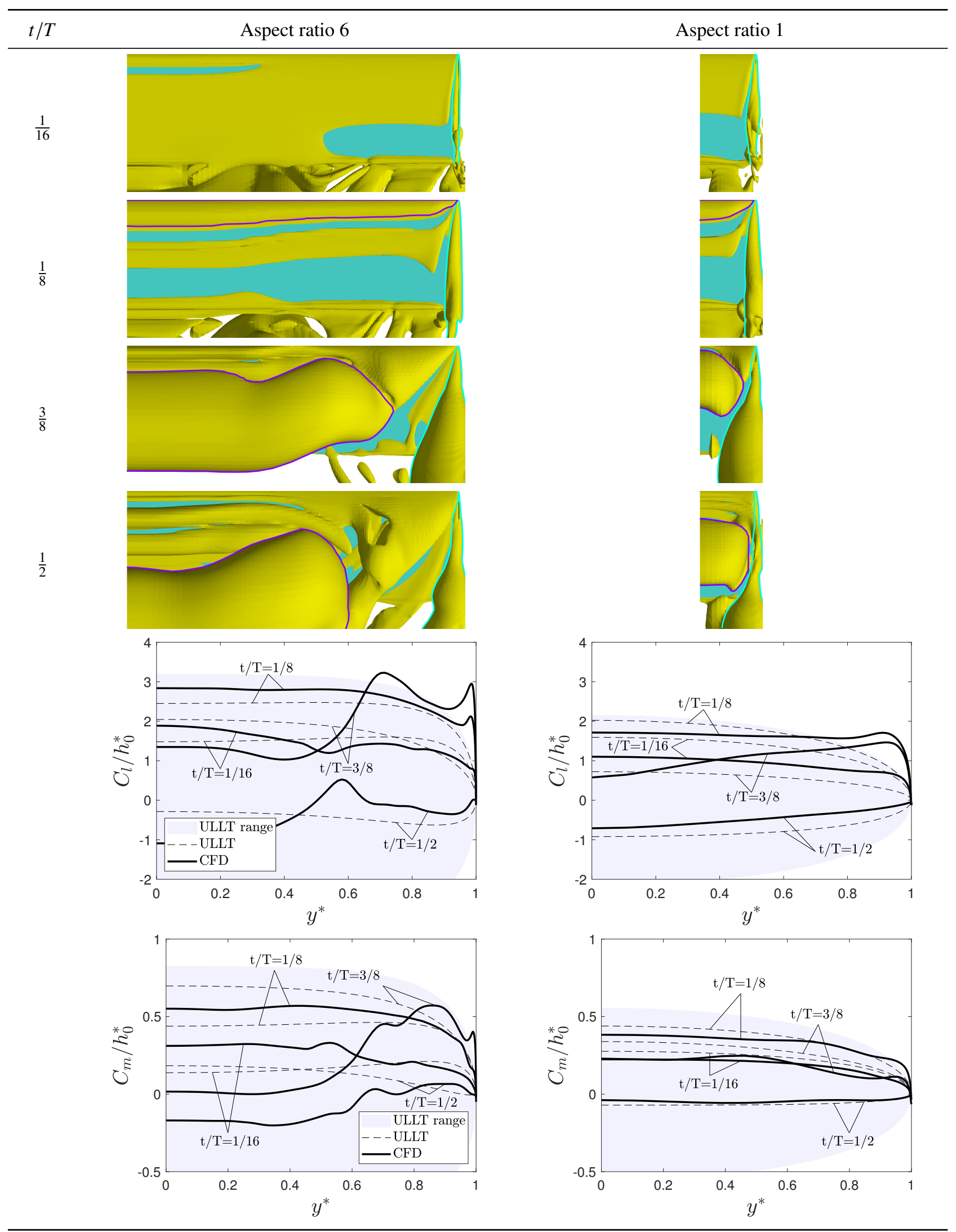

Fig. 10 Lift distributions, moment distributions and $\mathbf{Q}$ criterion iso-surfaces $(Q=1)$ for a wing oscillating in heave with amplitude $h_{0}^{*}=1$ at $k=0.4$. Moments are taken about the mid-chord. Major vortex structures are highlighted. 


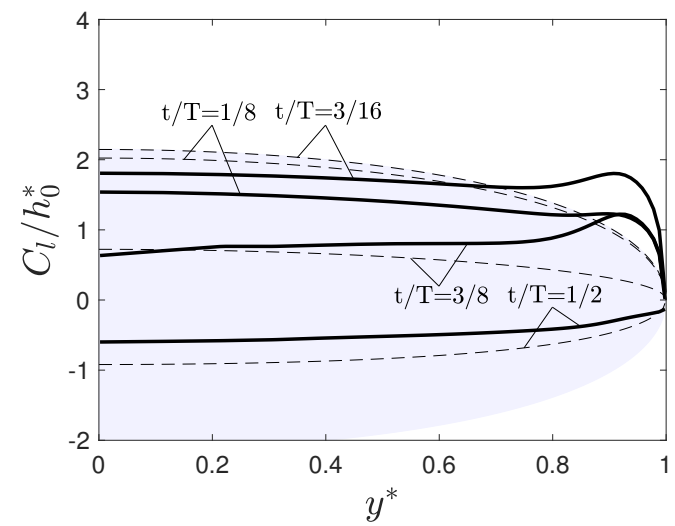

(a) Aspect ratio 1

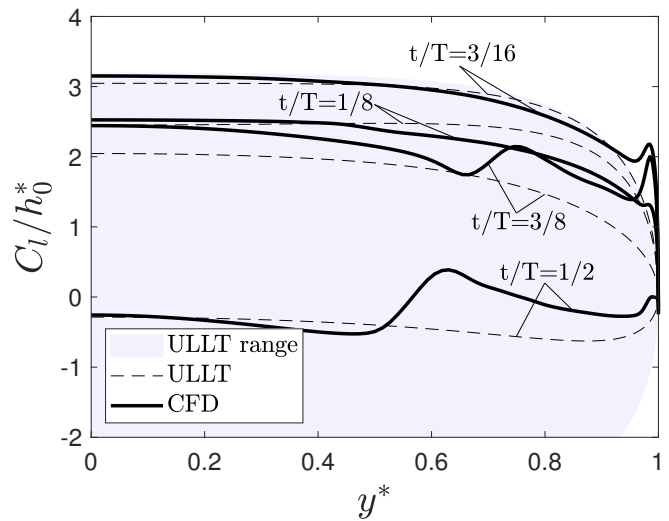

(b) Aspect ratio 6

Fig. $11 C_{l}$ distribution for rectangular wings oscillating in heave with amplitude $h_{0}^{*}=0.5$ at $k=0.4$.

At aspect ratio 6, the leading-edge vortex structure becomes more complex. This manifests itself in both the $C_{m}$ and $C_{l}$ distribution. At $t / T=1 / 8$, a nascent vortex is forming along almost the entire leading edge. By $t / T=3 / 8$ a large arch-type vortex structure has formed. The feet of the arch vortex stay connected to wing surface, with vortex structures extending to the very leading-edge corner of the wing. This arch vortex evolves with time, with the feet of the arch vortex moving downstream and towards the wing center. The arch-type vortex has a significant impact on the force distributions. At $t / T=1 / 16$, the $C_{l}$ and $C_{m}$ curves are relatively smooth, with some non-smoothness resulting due to the shedding of an LEV on the return stroke of the previous oscillation. They are still smooth at $t / T=1 / 8$ at the beginning of LEV formation, and less effected by the previous LEV. However, the large LEV arch vortex structure formed by $t / T=3 / 8$ has a significant impact. The impact on force distribution depends on the location of the arch vortex. Outside the arch vortex, near the wing tips, the influence of the arch-type vortex is to increase lift. Inside the arch vortex, lift is decreased, possibly resulting in premature negative lift coefficients compared to the non-LEV shedding case. The effect is strongest when the LEV is closest to the wing at $t / T=3 / 8$ compared to $t / T=1 / 2$. Near the wing tip, the force distribution from CFD is also influenced by separation at the wing tip. For a more detailed description of the vortex structures, see Visbal et al. [21].

Again, ULLT combined with LESP predicts that LEV formation will occur, but does not yield useful information about how the force distributions are impacted. The difference between the ULLT prediction and the CFD result at $t / T=1 / 16$ emphasizes how, despite the fact that ULLT predicts that $\mathcal{L}(y)<\mathcal{L}_{\text {crit }}$ across the entire wing span, the LEV shed in previous oscillations can impact the lift distribution on the wing.

Finally, the lift distributions for the $h_{0}^{*}=0.5$ cases are examined, shown in Fig. 11 The lift distributions are given at $t / T=\{1 / 8,3 / 16,3 / 8,1 / 2\}$, matching the time steps used to study the growth of the LEV structure shown in Fig. 8

The $C_{l}$ distribution for the aspect ratio 1 case is shown in Fig. 11(a), The lift distributions obtained from the CFD are similar to that for the higher amplitude $h_{0}^{*}=1$ case (see in Fig. 10p. The ULLT fails to predict the increased lift near 
the wing tip found in the CFD data. The ULLT combined with the LESP criterion predicts LEV shedding in the center of the wing (see Fig 6(b)], with the critical value of the leading edge suction parameter only just being exceeded. The effects of the LEV on the $C_{l}$ distribution appear to be negligible. Consequently, the error in the ULLT prediction of forces is a result of separation at the edges of the wing tip.

At aspect ratio 6, shown in Fig. 11(b), the inability of ULLT to capture the detail of the lift distribution obtained from CFD is again demonstrated. It is accurate at $t / T=1 / 8$, before LEV formation. However, like the $h_{0}^{*}=1$ case (see Fig. 10, the LEV arch-type vortex eventually creates a recognizable disturbance in the lift distribution that the ULLT cannot replicate. Due to the lower amplitude of oscillation, ULLT combined with the LESP criterion predicts that the critical value of leading edge suction is exceeded to a lesser extent in comparison to the larger amplitude case. As a result, the resulting LEV is weaker, and the impact of the LEV of the lift distribution is diminished. The LESP criterion cannot reflect the 3D evolution of the vortex structure, but the extent to which the critical LESP is exceeded does broadly predict the extent to which the predicted force distribution deviates from the true value.

\section{Conclusions}

An Unsteady Lifting-Line Theory (ULLT) for finite wings oscillating in heave was presented, including a means to find both whole wing forces, wing force distribution and leading edge suction distribution. This inviscid, small-amplitude theory was then compared to CFD results for low Reynolds number regime cases, including ones with large amplitude oscillations leading to leading edge vortex shedding.

ULLT predicted the whole wing lift and moments for rectangular wings with sufficient accuracy for many applications. For small amplitude oscillations, the prediction was very good. As the amplitude increased, aerodynamic non-linearities caused deviation from the assumed sinusoidal form of the forces with respect to time. The ULLT assumes a sinusoidal response since the physics it models is simplified in comparison to CFD. However, these deviations from the sinusoidal form reduced significantly as aspect ratio decreased - 3D effects stabilized the leading edge vortex structure.

For small amplitude oscillation, the ULLT predicted the load distribution well. As oscillation amplitude increased, aerodynamic non-linearities caused a poor prediction of the wing load distribution. The prediction became worse as the amplitude increased.

The ULLT, combined with the leading edge suction criterion, could be used to predict leading edge vortex shedding, but not the spanwise location of the shedding, or the exact timing. Consequently, it acts as a coarse indicator of the usefulness of of the ULLT predicted wing load distributions. This indicator is less useful at very low aspect ratio due to the importance of vortical structures caused by separation at the wing tips.

Broadly, we find that inviscid ULLT is indeed very useful in approximate prediction of aerodynamic forces on oscillating wings in the low Reynolds number regime. Potential enhancements to the ULLT which are the subject of future research include, the use of a higher-fidelity inner solution such as Taha and Rezaei's viscous extension to the 
oscillating aerofoil problem [69], and the use of discrete-vortex methods to model separated flow features [36, 70].

\section{Funding Sources}

The authors gratefully acknowledge the support of the UK Engineering and Physical Sciences Research Council (EPSRC) through a DTA scholarship and grant EP/R008035. We also thank the Cirrus UK National Tier-2 HPC service at EPCC (http://www.cirrus.ac.uk) for a computing grant, and the Japan Student Services Organization (JASSO) for their generous financial support.

\section{References}

[1] McCroskey, W. J., “The Phenomenon of Dynamic Stall,” Tech. rep., NASA, Mar. 1981. URL https://ntrs.nasa.gov/ archive/nasa/casi.ntrs.nasa.gov/19810011501.pdf

[2] Carr, L., "Progress in analysis and prediction of dynamic stall," Journal of Aircraft, Vol. 25, No. 1, 1988, pp. 6-17.

[3] Carr, L. W., Platzer, M. F., Chandrasekhara, M. S., and Ekaterinaris, J., "Experimental and Computational Studies of Dynamic Stall," Numerical and Physical Aspects of Aerodynamic Flows IV, edited by T. Cebeci, Springer Berlin Heidelberg, 1990, pp. $239-256$.

[4] Ekaterinaris, J. A., and Platzer, M. F., "Computational prediction of airfoil dynamic stall," Progress in aerospace sciences, Vol. 33, No. 11, 1998, pp. 759-846.

[5] Andreu Angulo, I., and Ansell, P. J., "Influence of aspect ratio on dynamic stall of a finite wing," AIAA journal, 2019, pp. $2722-2733$.

[6] Visbal, M. R., and Garmann, D. J., "Dynamic stall of a finite-aspect-ratio wing," AIAA Journal, Vol. 57, No. 3, 2019, pp. 962-977.

[7] Visbal, M. R., and Garmann, D. J., "Effect of sweep on dynamic stall of a pitching finite-aspect-ratio wing," AIAA Journal, Vol. 57, No. 8, 2019, pp. 3274-3289.

[8] Costes, M., Richez, F., Pape, A. L., and Gavériaux, R., "Numerical investigation of three-dimensional effects during dynamic stall," Aerospace Science and Technology, Vol. 47, 2015, pp. 216-237. https://doi.org/10.1016/j.ast.2015.09.025.

[9] Mulleners, K., Mancini, P., and Jones, A. R., "Flow development on a flat-plate wing subjected to a streamwise acceleration," AIAA Journal, Vol. 55, No. 6, 2017, pp. 2118-2122.

[10] Mancini, P., Manar, F., Granlund, K., Ol, M. V., and Jones, A. R., "Unsteady aerodynamic characteristics of a translating rigid wing at low Reynolds number,” Physics of Fluids, Vol. 27, No. 12, 2015, p. 123102.

[11] DeVoria, A. C., and Mohseni, K., "On the mechanism of high-incidence lift generation for steadily translating low-aspect-ratio wings," Journal of Fluid Mechanics, Vol. 813, 2017, pp. 110-126. 
[12] Jantzen, R. T., Taira, K., Granlund, K., and Ol, M. V., "Vortex dynamics around pitching plates," Physics of Fluids, Vol. 26, No. 5, 2014, p. 053606.

[13] Hord, K., and Lian, Y., "Leading Edge Vortex Circulation Development on Finite Aspect Ratio Pitch-Up Wings," AIAA Journal, 2016, pp. 2755-2767.

[14] Yilmaz, T. O., and Rockwell, D., "Flow structure on finite-span wings due to pitch-up motion,” Journal of Fluid Mechanics, Vol. 691, 2012, pp. 518-545.

[15] Yilmaz, T., Ol, M., and Rockwell, D., "Scaling of flow separation on a pitching low aspect ratio plate," Journal of Fluids and Structures, Vol. 26, No. 6, 2010, pp. 1034-1041.

[16] Visbal, M. R., "Unsteady flow structure and loading of a pitching low-aspect-ratio wing," Physical Review Fluids, Vol. 2, No. 2, 2017, p. 024703.

[17] Green, M. A., Rowley, C. W., and Smits, A. J., “The unsteady three-dimensional wake produced by a trapezoidal pitching panel," Journal of Fluid Mechanics, Vol. 685, 2011, pp. 117-145.

[18] Calderon, D. E., Wang, Z., Gursul, I., and Visbal, M., "Volumetric measurements and simulations of the vortex structures generated by low aspect ratio plunging wings," Physics of Fluids, Vol. 25, No. 6, 2013, p. 067102.

[19] Calderon, D., Cleaver, D., Gursul, I., and Wang, Z., "On the absence of asymmetric wakes for periodically plunging finite wings," Physics of Fluids, Vol. 26, No. 7, 2014, pp. 349-376.

[20] Calderon, D., Wang, Z., and Gursul, I., "Lift-enhancing vortex flows generated by plunging rectangular wings with small amplitude," AIAA journal, Vol. 51, No. 12, 2013, pp. 2953-2964.

[21] Visbal, M. R., Yilmaz, T. O., and Rockwell, D., “Three-dimensional vortex formation on a heaving low-aspect-ratio wing: computations and experiments," Journal of Fluids and Structures, Vol. 38, 2013, pp. 58-76.

[22] Yilmaz, T. O., and Rockwell, D., “Three-dimensional flow structure on a maneuvering wing," Experiments in Fluids, Vol. 48, No. 3, 2010, pp. 539-544.

[23] Fishman, G., Wolfinger, M., and Rockwell, D., "The structure of a trailing vortex from a perturbed wing," Journal of Fluid Mechanics, Vol. 824, 2017, pp. 701-721.

[24] Ozen, C. A., and Rockwell, D., “Three-dimensional vortex structure on a rotating wing," Journal of Fluid Mechanics, Vol. 707, 2012, pp. 541-550.

[25] Carr, Z. R., Chen, C., and Ringuette, M. J., "Finite-span rotating wings: three-dimensional vortex formation and variations with aspect ratio," Experiments in fluids, Vol. 54, No. 2, 2013, pp. 1-26.

[26] Carr, Z. R., DeVoria, A. C., and Ringuette, M. J., “Aspect-ratio effects on rotating wings: circulation and forces,” Journal of Fluid Mechanics, Vol. 767, 2015, pp. 497-525. 
[27] Medina, A., and Jones, A. R., "Leading-edge vortex burst on a low-aspect-ratio rotating flat plate," Physical Review Fluids, Vol. 1, No. 4, 2016, p. 044501.

[28] Beals, N., and Jones, A. R., "Lift production by a passively flexible rotating wing," AIAA Journal, Vol. 53, No. 10, 2015 , pp. 2995-3005.

[29] Venkata, S. K., and Jones, A. R., "Leading-edge vortex structure over multiple revolutions of a rotating wing," Journal of aircraft, Vol. 50, No. 4, 2013, pp. 1312-1316.

[30] Perrotta, G., and Jones, A. R., “Unsteady forcing on a flat-plate wing in large transverse gusts,” Experiments in Fluids, Vol. 58, No. 8, 2017, p. 101.

[31] Corkery, S., Babinsky, H., and Harvey, J., "On the development and early observations from a towing tank-based transverse wing-gust encounter test rig," Experiments in Fluids, Vol. 59, No. 9, 2018, p. 135.

[32] Biler, H., Badrya, C., and Jones, A. R., "Experimental and computational investigation of transverse gust encounters," AIAA Journal, Vol. 57, No. 11, 2019, pp. 4608-4622.

[33] Theodorsen, T., "General Theory of Aerodynamic instability and the mechanism of flutter," Tech. Rep. 496, NACA, 1935.

[34] Ramesh, K., Gopalarathnam, A., Edwards, J. R., Ol, M. V., and Granlund, K., "An unsteady airfoil theory applied to pitching motions validated against experiment and computation," Theoretical and Computational Fluid Dynamics, Vol. 27, No. 6, 2013, pp. 843-864. https://doi.org/10.1007/s00162-012-0292-8

[35] Yan, Z., Taha, H. E., and Hajj, M. R., "Geometrically-exact unsteady model for airfoils undergoing large amplitude maneuvers," Aerospace Science and Technology, Vol. 39, 2014, pp. 293-306. https://doi.org/10.1016/j.ast.2014.09.021

[36] Ramesh, K., Gopalarathnam, A., Granlund, K., Ol, M. V., and Edwards, J. R., "Discrete-vortex method with novel shedding criterion for unsteady aerofoil flows with intermittent leading-edge vortex shedding," Journal of Fluid Mechanics, Vol. 751, 2014, pp. 500-538. https://doi.org/10.1017/jfm.2014.297

[37] Katz, J., "A discrete vortex method for the non-steady separated flow over an airfoil,” Journal of Fluid Mechanics, Vol. 102, No. -1, 1981, p. 315. https://doi.org/10.1017/s0022112081002668

[38] Moored, K. W., "Unsteady three-dimensional boundary element method for self-propelled bio-inspired locomotion,” Computers \& Fluids, Vol. 167, 2018, pp. 324-340.

[39] Katz, J., and Plotkin, A., Low Speed Aerodynamics, $2^{\text {nd }}$ ed., Cambridge University Press, 2001.

[40] Murua, J., Palacios, R., and Graham, J. M. R., "Applications of the unsteady vortex-lattice method in aircraft aeroelasticity and flight dynamics,” Progress in Aerospace Sciences, Vol. 55, 2012, pp. 46-72. https://doi.org/10.1016/j.paerosci.2012.06.001.

[41] Smyth, A. S., Young, A. M., and Mare, L. D., "The Effect of 3D Geometry on Unsteady Gust Response, Using a Vortex Lattice Model," AIAA Scitech 2019 Forum, American Institute of Aeronautics and Astronautics, 2019. https://doi.org/10.2514/6.20190899 
[42] Hirato, Y., Shen, M., Gopalarathnam, A., and Edwards, J. R., "Vortex-Sheet Representation of Leading-Edge Vortex Shedding from Finite Wings," Journal of Aircraft, 2019, pp. 1-15. https://doi.org/10.2514/1.c035124.

[43] Willis, D. J., Peraire, J., and White, J. K., “A combined pFFT-multipole tree code, unsteady panel method with vortex particle wakes," International Journal for Numerical Methods in Fluids, Vol. 53, No. 8, 2007, pp. 1399-1422. https: //doi.org/10.2514/6.2005-854

[44] Leishman, G. J., Principles of Helicopter Aerodynamics, Cambridge University Press, 2006.

[45] Prandtl, L., “Applications of Modern Hydrodynamics to Aeronautics,” Tech. rep., NACA, 1923. Rep. 116.

[46] Van Dyke, M., "Lifting-line theory as a singular-perturbation problem," Journal of Applied Mathematics and Mechanics, Vol. 28, No. 1, 1964, pp. 90-102. https://doi.org/10.1016/0021-8928(64)90134-0

[47] James, E. C., "Lifting-line theory for an unsteady wing as a singular perturbation problem," Journal of Fluid Mechanics, Vol. 70, No. 04, 1975, p. 753. https://doi.org/10.1017/s0022112075002339

[48] Holten, T. V., "Some notes on unsteady lifting-line theory," Journal of Fluid Mechanics, Vol. 77, No. 03, 1976 , p. 561. https://doi.org/10.1017/s0022112076002255.

[49] Ahmadi, A. R., and Widnall, S. E., "Unsteady lifting-line theory as a singular perturbation problem," Journal of Fluid Mechanics, Vol. 153, 1985, p. 59. https://doi.org/10.1017/s0022112085001148

[50] Sclavounos, P. D., “An unsteady lifting-line theory,” Journal of Engineering Mathematics, Vol. 21, No. 3, 1987 , pp. $201-226$. https://doi.org/10.1007/bf00127464.

[51] Guermond, J.-L., and Sellier, A., “A unified unsteady lifting-line theory,” Journal of Fluid Mechanics, Vol. 229, 1991 , p. 427. https://doi.org/10.1017/s0022112091003099

[52] Jones, R. T., "The unsteady lift of a wing of finite aspect ratio," Tech. rep., National Advisory Committee for Aeronautics, 1940.

[53] Devinant, P., "An approach for unsteady lifting-line time-marching numerical computation,” International Journal for Numerical Methods in Fluids, Vol. 26, No. 2, 1998, pp. 177-197. https://doi.org/10.1002/(sici)1097-0363(19980130)26:2<177::aidfld633>3.3.co;2-g.

[54] Ramesh, K., Monteiro, T. P., Silvestre, F. J., Bernardo, A., aes Neto, G., de Souza Siqueria Versiani, T., and da Silva, R. G. A., "Experimental and Numerical Investigation of Post-Flutter Limit Cycle Oscillations on a Cantilevered Flat Plate," International Forum on Aeroelasticity and Structural Dynamics 2017, 2017. URL http://eprints.gla.ac.uk/154722/.

[55] Boutet, J., and Dimitriadis, G., "Unsteady Lifting Line Theory Using the Wagner Function for the Aerodynamic and Aeroelastic Modeling of 3D Wings," Aerospace, Vol. 5, No. 3, 2018, p. 92. https://doi.org/10.3390/aerospace5030092

[56] Sugar-Gabor, O., "A general numerical unsteady non-linear lifting line model for engineering aerodynamics studies," The Aeronautical Journal, Vol. 122, No. 1254, 2018, pp. 1199-1228. https://doi.org/10.1017/aer.2018.57 
[57] Bird, H. J. A., Otomo, S., Ramesh, K., and Viola, I. M., “A Geometrically Non-Linear Time-Domain Unsteady Lifting-Line Theory," AIAA Scitech 2019 Forum, American Institute of Aeronautics and Astronautics, 2019. https://doi.org/10.2514/6.20191377 .

[58] Bird, H. J. A., and Ramesh, K., "Unsteady lifting-line theory and the influence of wake vorticity on aerodynamic loads," Theoretical and Computational Fluid Dynamics, 2021. https://doi.org/10.1007/s00162-021-00578-8

[59] McGowan, G. Z., Granlund, K., Ol, M. V., Gopalarathnam, A., and Edwards, J. R., "Investigations of Lift-Based PitchPlunge Equivalence for Airfoils at Low Reynolds Numbers," AIAA Journal, Vol. 49, No. 7, 2011, pp. $1511-1524$. https://doi.org/10.2514/1.j050924

[60] Elfering, K. H., and Granlund, K. O., "Lift Equivalence and Cancellation for Airfoil Surge-Pitch-Plunge Oscillations," AIAA Journal, 2020, pp. 1-15. https://doi.org/10.2514/1.j059068

[61] Bird, H. J. A., and Ramesh, K., "Theoretical and computational studies of a rectangular finite wing oscillating in pitch and heave," 6th European Conference on Computational Mechanics (ECCM 6) 7th European Conference on Computational Fluid Dynamics (ECFD 7), International Centre for Numerical Methods in Engineering, 2018.

[62] Olver, F. W., Lozier, D. W., Boisvert, R. F., and Clark, C. W., NIST Handbook of Mathematical Functions Paperback and CD-ROM, Cambridge University Press, 2010.

[63] Ramesh, K., Granlund, K., Ol, M. V., Gopalarathnam, A., and Edwards, J. R., "Leading-edge flow criticality as a governing factor in leading-edge vortex initiation in unsteady airfoil flows," Theoretical and Computational Fluid Dynamics, Vol. 32, No. 2, 2018, pp. 109-136. https://doi.org/10.1007/s00162-017-0442-0.

[64] Hirato, Y., Shen, M., Gopalarathnam, A., and Edwards, J. R., "Flow Criticality Governs Leading-Edge-Vortex Initiation on Finite Wings in Unsteady Flow," Journal of Fluid Mechanics, 2020. https://doi.org/10.1017/jfm.2020.896, submitted for publication.

[65] Ramesh, K., "On the leading-edge suction and stagnation-point location in unsteady flows past thin aerofoils," Journal of Fluid Mechanics, Vol. 886, 2020. https://doi.org/10.1017/jfm.2019.1070

[66] Weller, H. G., Tabor, G., Jasak, H., and Fureby, C., "A tensorial approach to computational continuum mechanics using object-oriented techniques," Computers in Physics, Vol. 12, No. 6, 1998, p. 620. https://doi.org/10.1063/1.168744

[67] Spalart, P., and Allmaras, S., “A one-equation turbulence model for aerodynamic flows," 30th Aerospace Sciences Meeting and Exhibit, American Institute of Aeronautics and Astronautics, 1992. https://doi.org/10.2514/6.1992-439. AIAA Paper 1992-0439.

[68] Hunt, J. C. R., Wray, A. A., and Moin, P., "Eddies, streams, and convergence zones in turbulent flows," Proceedings of the 1988 Summer Program, Center for Turbulence Research, Stanford University, 1988, pp. 193-208. URL https: //ntrs.nasa.gov/citations/19890015184 
[69] Taha, H., and Rezaei, A. S., "Viscous extension of potential-flow unsteady aerodynamics: the lift frequency response problem," Journal of Fluid Mechanics, Vol. 868, 2019, pp. 141-175. https://doi.org/10.1017/jfm.2019.159

[70] Bird, H. J. A., Ramesh, K., Ōtomo, S., and Viola, I. M., "Leading Edge Vortex Formation on Finite Wings Using Vortex Particles," AIAA Scitech 2021 Forum, American Institute of Aeronautics and Astronautics, 2021. https://doi.org/10.2514/6.2021-1196 